\title{
Note: An improved low-frequency correction technique for piezoelectric force sensors in high-speed nanopositioning systems
}

Yuen K. Yong and, and Andrew J. Fleming

Citation: Review of Scientific Instruments 88, 046105 (2017); doi: 10.1063/1.4981530

View online: http://dx.doi.org/10.1063/1.4981530

View Table of Contents: http://aip.scitation.org/toc/rsi/88/4

Published by the American Institute of Physics

\section{Articles you may be interested in}

Invited Review Article: Tip modification methods for tip-enhanced Raman spectroscopy (TERS) and colloidal probe technique: A 10 year update (2006-2016) review

Review of Scientific Instruments 88, 031101 (2017); 10.1063/1.4978929

Note: Development of a new Bridgman-type high pressure cell for accurate dielectric measurements Review of Scientific Instruments 88, 046104 (2017); 10.1063/1.4980154

Development of a bio-magnetic measurement system and sensor configuration analysis for rats

Review of Scientific Instruments 88, 044704 (2017); 10.1063/1.4979071

Note: Manganin foil sensor for small uniaxial stress

Review of Scientific Instruments 88, 046106 (2017); 10.1063/1.4981247

Improved design for a low temperature scanning tunneling microscope with an in situ tip treatment stage Review of Scientific Instruments 88, 043702 (2017); 10.1063/1.4979928

Instrumentation and control of harmonic oscillators via a single-board microprocessor-FPGA device

Review of Scientific Instruments 88, 045108 (2017); 10.1063/1.4979971

STEM CAREER WEBINARS

on networking, interviewing, conferences, presenting...

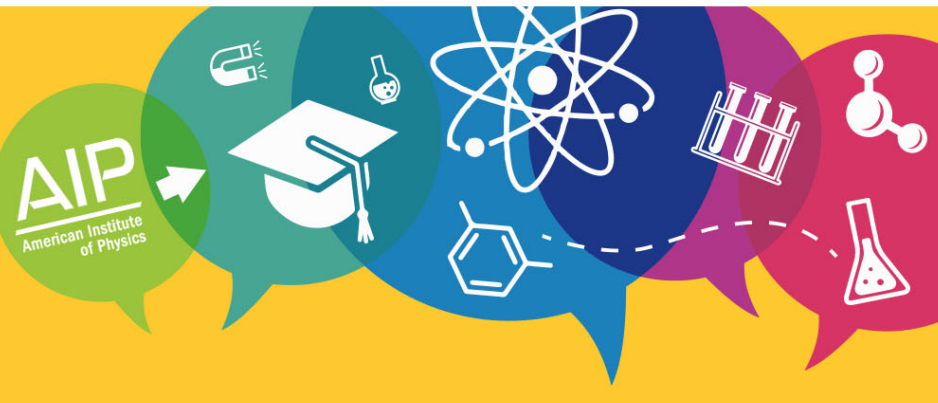




\title{
Note: An improved low-frequency correction technique for piezoelectric force sensors in high-speed nanopositioning systems
}

\author{
Yuen K. Yonga) and Andrew J. Fleming ${ }^{\text {b) }}$ \\ School of Electrical Engineering and Computing, University of Newcastle, Callaghan, NSW, Australia
}

(Received 25 October 2016; accepted 6 April 2017; published online 21 April 2017)

\begin{abstract}
Piezoelectric force and position sensors provide high sensitivity but are limited at low frequencies due to their high-pass response which complicates the direct application of integral control. To overcome this issue, an additional sensor or low-frequency correction method is typically employed. However, these approaches introduce an additional first-order response that must be higher than the high-pass response of the piezo and interface electronics. This article describes a simplified method for lowfrequency correction that uses the piezoelectric sensor as an electrical component in a filter circuit. The resulting response is first-order, rather than second-order, with a cut-off frequency equal to that of a buffer circuit with the same input resistance. The proposed method is demonstrated to allow simultaneous damping and tracking control of a high-speed vertical nanopositioning stage. Published by AIP Publishing. [http://dx.doi.org/10.1063/1.4981530]
\end{abstract}

Piezoelectric sensors have been widely used for vibration control in nanopositioning systems ${ }^{1,2}$ and smart structures. ${ }^{3}$ These sensors exhibit some outstanding properties such as wide bandwidth, high sensitivity, and low noise at high frequencies. Compared to capacitive, inductive, and optical sensors, piezoelectric sensors are extremely compact and can be bonded directly to high-speed nanopositioners for vibration control, ${ }^{4}$ and in some cases, tracking control. ${ }^{2,4}$ Piezoelectric sensors also have the benefit of simple interface circuitry and low cost.

Piezoelectric sensors have been used to damp the resonances of nanopositioning red for tracking control. ${ }^{5,6}$ Although piezoelectric sensors have excellent AC properties, they are not suitable for use at DC due to their high-pass response which can destabilize a standard integral control loop. ${ }^{2,4}$

A common approach for dealing with the high-pass response of piezoelectric sensors is the low-frequency bypass method. ${ }^{2,4}$ In this method, the low-frequency component of the piezoelectric sensor voltage is substituted with either an auxillary DC sensor or a scaled version of the actuator input voltage. A disadvantage of this method is that the complementary filters used in this technique must have a significantly higher cut-off frequency than the sensor. For example, if a buffered piezoelectric sensor has a cut-off frequency of $0.1 \mathrm{~Hz}$, the complimentary filter poles must be higher in frequency than $1 \mathrm{~Hz}$ to avoid a significant phase error due to the high-pass response of the sensor. Therefore, present methods cannot fully utilize the available response of the sensor.

In this article, a simplified method for low-frequency correction is described that eliminates the need for additional complementary filters by using the piezoelectric sensor as a filter component. The impedance of the piezoelectric sensor is combined with an external resistor and buffer to create

\footnotetext{
a)yuenkuan.yong@newcastle.edu.au

b) andrew.fleming@newcastle.edu.au
}

a complementary filter with an arbitrary cut-off frequency and first-order response. The resulting cut-off frequency of the complementary filter is equal to that of a buffer circuit with the same input resistance. The response is also firstorder, rather than second-order, which simplifies the control design. The proposed approach is demonstrated by implementing an integral force feedback (IFF) controller which damps the resonance and provides position control. ${ }^{2}$

The proposed low-frequency correction technique is demonstrated on the flexure-guided vertical nanopositioner shown in Fig. 1. A piezoelectric stack actuator with an integrated force sensor is used to drive the nanopositioner vertically. The sensor is electrically isolated from the actuator by a $0.5-\mathrm{mm}$ ceramic plate which also reduces the transmission of lateral strain between the actuator and sensor (due to Poisson coupling in the actuator during elongation). The piezoelectric sensor measures the force applied to the moving stage which is used as a feedback variable for both tracking and damping control.

The simplified electrical model of a piezoelectric force sensor is shown in Fig. 2. The induced voltage $V_{p}$ is high-pass filtered by the capacitance $C_{p}$ and input resistance $R_{i n} .{ }^{2}$ The cut-off frequency is $f_{c}=1 /\left(2 \pi R_{i n} C_{p}\right)$. The circuit used in this

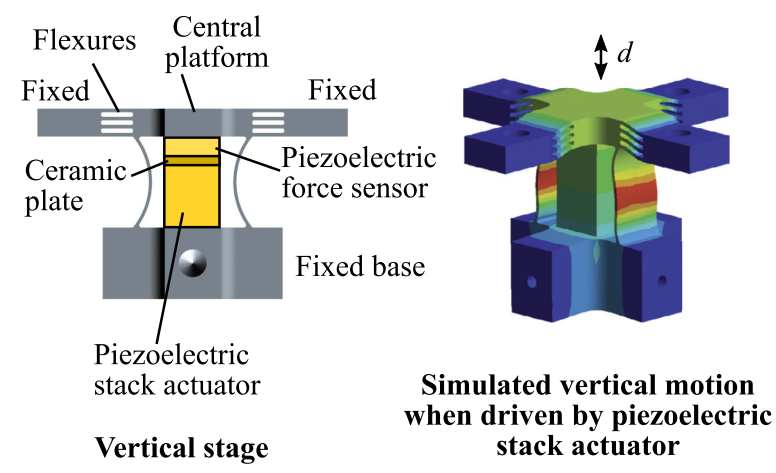

FIG. 1. Flexure-guided vertical nanopositioner driven by a piezoelectric stack actuator with an integrated force sensor. 


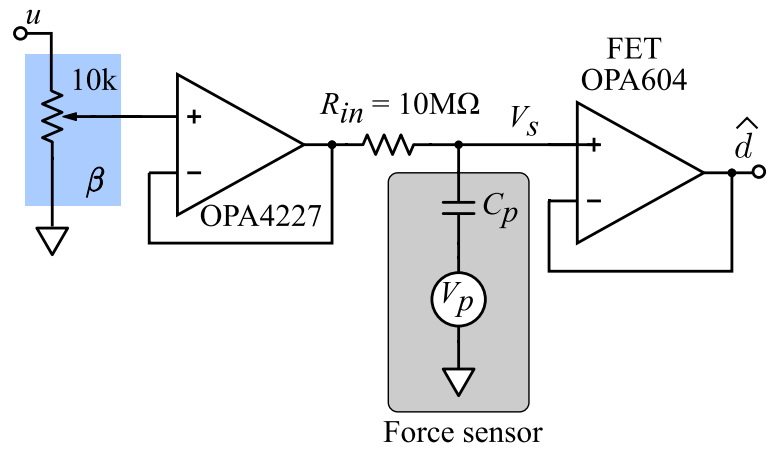

FIG. 2. Low-frequency correction circuit diagram. The piezoelectric force sensor voltage is modeled by a voltage source and capacitance $C_{p}$.

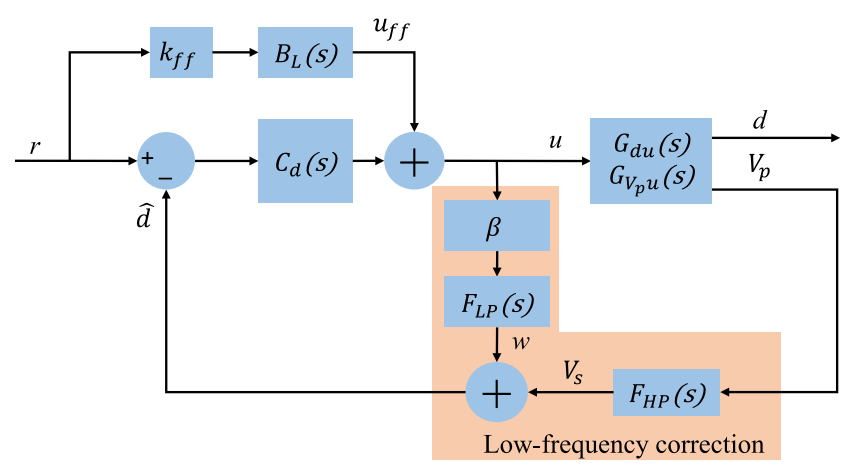

FIG. 3. Block diagram of the closed-loop system with integral force feedback and a feedforward input. The low-frequency correction method is represented by the shaded region.

work has an input resistance of $R_{i n}=10 \mathrm{M} \Omega$ and the capacitance $C_{p}$ is $158 \mathrm{nF}$ which results in a $0.1-\mathrm{Hz}$ cut-off frequency. The transfer function from $V_{p}$ to $V_{s}$ (measured voltage across the piezoelectric force sensor) is

$$
F_{H P}(s)=\frac{V_{s}(s)}{V_{p}(s)}=\frac{s}{s+1 /\left(R_{i n} C_{p}\right)} .
$$

This high-pass characteristic destabilizes a control system with integral action. Fig. 3 illustrates the standard low-frequency correction technique employed in a feedback

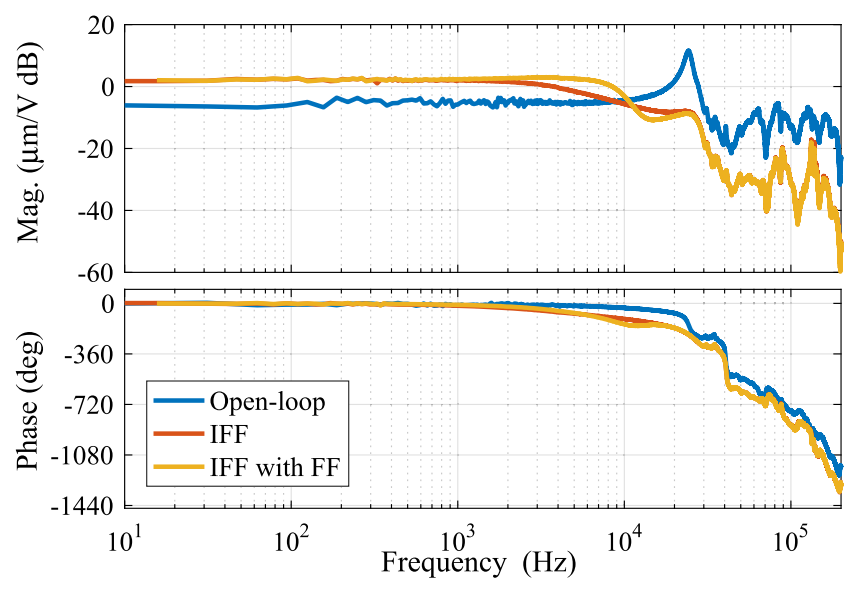

FIG. 4. Measured frequency responses of the vertical stage in open-loop $G_{d u}$, closed-loop with IFF control $G_{d r}$, and closed-loop with IFF and feedforward input $G_{\widehat{d} r}^{f f}$.

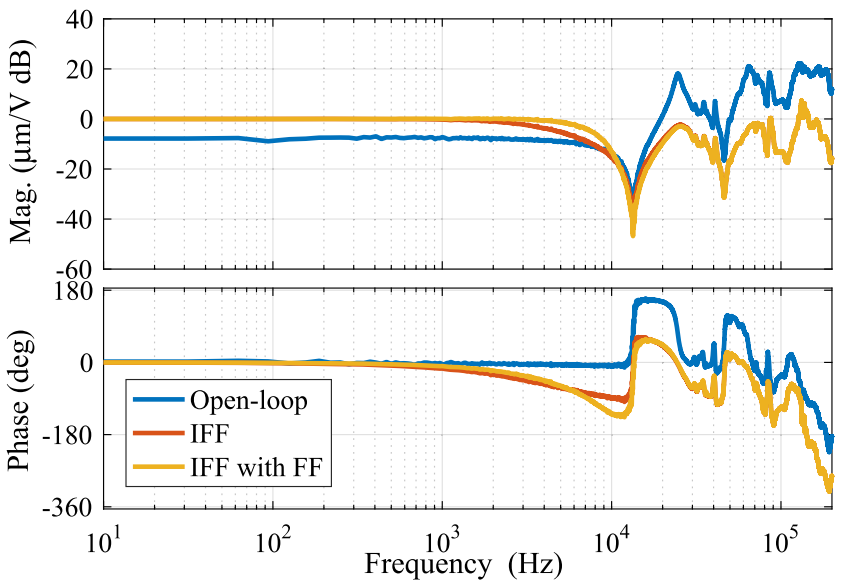

FIG. 5. Measured frequency responses of the vertical stage in open-loop $\left(G_{\widehat{d} u}\right)$, closed-loop with IFF control $\left(G_{\widehat{d} r}\right)$, and closed-loop with IFF and feedforward input $G_{\widehat{d} r}^{f f}$.

control loop. ${ }^{2}$ A low-pass filter $F_{L P}$ which is complementary to $F_{H P}$ is placed in the tracking loop, that is,

$$
F_{L P}(s)=\frac{1 /\left(R_{i n} C_{p}\right)}{s+1 /\left(R_{i n} C_{p}\right)} .
$$

The constant gain $\beta$ is used to equalize the DC sensitivity of the two signal paths, that is, $\beta=G_{V_{p} u}(0)$. Once $\beta$ is correctly chosen, the low-frequency response from $u$ to $\widehat{d}$ is

$$
\frac{\widehat{d}}{u}=G_{V_{p} u}(0)\left(F_{L P}(s)+F_{H P}(s)\right)=G_{V_{p} u}(0) .
$$

Therefore, the low-frequency dynamics due to $F_{H P}(s)$ have been eliminated.

In the previous work, ${ }^{2,4}$ the complimentary filters are implemented by additional filters. However, it can be observed that the shaded area in Fig. 3 can be implemented by the simple analog network in Fig. 2. In this circuit, the source impedance of the piezoelectric sensor is utilized to implement both $F_{L P}(s)$ and $F_{H P}(s)$ where the transfer functions are described in Eqs. (1) and (2). This approach results in a single low-frequency pole with matched cut-off frequencies that are independent of the component values.

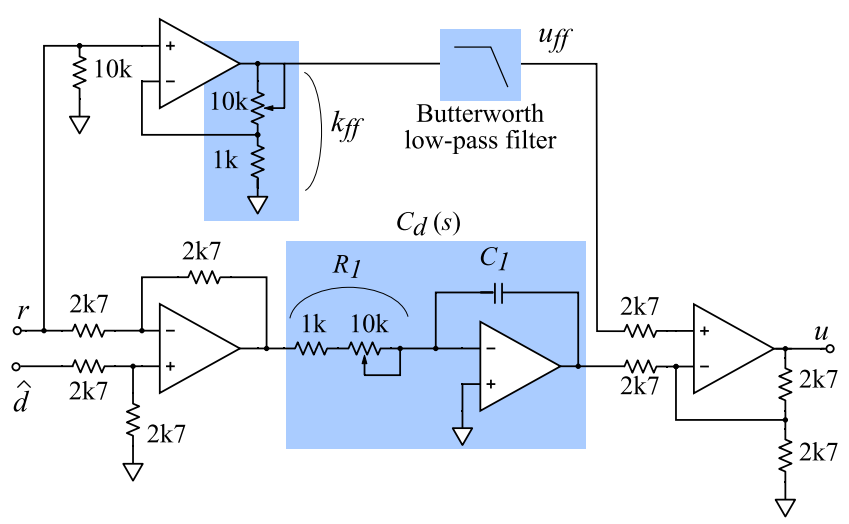

FIG. 6. Analog implementation of the IFF controller. The bottom signal path implements the summer and integrator (which are both inverting). The top signal path implements a feedforward controller that can be used to improve the bandwidth. 

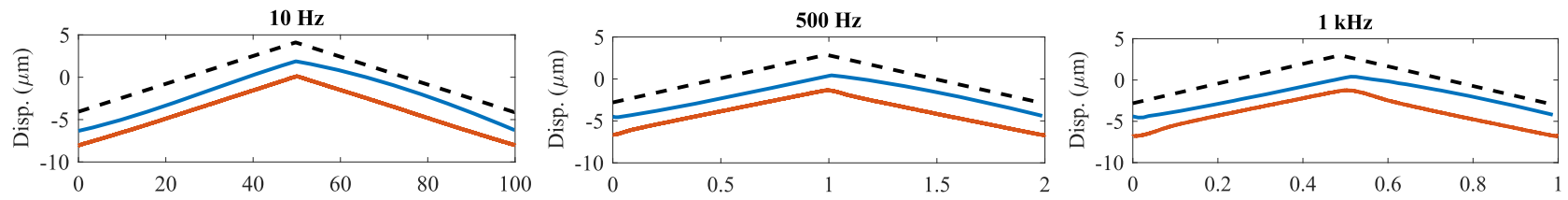

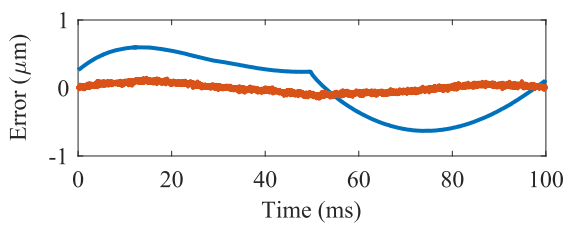

(a) rms err.: $\mathrm{OL}=417.4 \mathrm{~nm}$. $\mathrm{CL}=65.1 \mathrm{~nm}$.

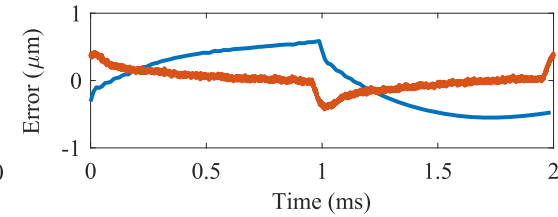

(b) $\mathrm{rms}$ err.: $\mathrm{OL}=410 \mathrm{~nm}$. $\mathrm{CL}=146.8 \mathrm{~nm}$.

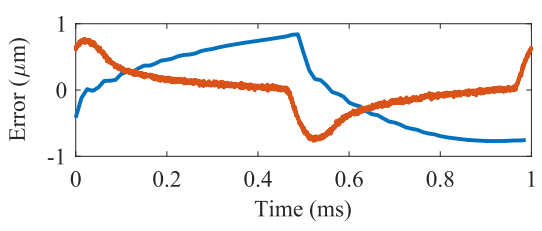

(c) $\mathrm{rms}$ err.: $\mathrm{OL}=563.5 \mathrm{~nm}$. $\mathrm{CL}=316.2 \mathrm{~nm}$.

FIG. 7. Open- (blue) and closed-loop (red) tracking performance of the vertical stage. Closed-loop plots are offset from the reference signal (black) for clarity sake. At $10 \mathrm{~Hz}$, the stage is forced to track a full range triangular input. At $500 \mathrm{~Hz}$ and $1 \mathrm{kHz}$, the reference inputs are reduced to $70 \%$ full range to avoid exceeding the current limit of the voltage amplifier.

To demonstrate the effectiveness of the low-frequency correction technique, the corrected signal $\widehat{d}$ is used for damping and tracking control. The vertical nanopositioning device is considered as a single-input two-output (SITO) system where the input $u$ is the applied voltage and the outputs are the measured displacement $d$ and the corrected piezoelectric force sensor voltage $\widehat{d}$ as shown in Fig. 3. The measured open-loop frequency responses (using a Polytec PSV-300 laser vibrometer) from $u$ to $d$ and $u$ to $\widehat{d}$ are plotted in Figs. 4 and 5, respectively. Due to the force sensor, ${ }^{2} G_{\widehat{d} u}$ exhibits an interlaced zero-pole pattern and the phase response lies between $0^{\circ}$ and $180^{\circ}$. Such systems are classified as negative imaginary systems and have guaranteed stability with an integral controller. ${ }^{7}$

An integral force feedback (IFF) controller suppresses the resonance modes and provides tracking control. The IFF loop consists of the plant $G_{\widehat{d} u}$ and an integrator $C_{d}=K_{i} / s$ as shown in Fig. 3. The closed-loop transfer function from $r$ to $d$ is

$$
G_{d r}=\frac{C_{d} G_{d u}}{1+C_{d} G_{\widehat{d} u}} .
$$

Due to the simplicity of the control loop, analog implementation is desirable for avoiding quantization noise and sampling delay. The control circuit diagram is shown in Fig. 6. An integral gain of $K_{i}=53850$ was found to achieve maximum damping. The component values were $R_{1}=1.85 \mathrm{k} \Omega$ and $C_{1}=10 \mathrm{nF}$.

The measured closed-loop frequency responses are illustrated in Figs. 4 and 5, respectively. The IFF controller effectively suppresses the resonant peak of the system while maintaining a gain and phase margin of $5 \mathrm{~dB}$ and $77.5^{\circ}$, respectively.

For tracking, the displacement is approximately proportional to force ${ }^{2}$ up until the frequency of the first system zero $f_{z}$, which occurs at $13.4 \mathrm{kHz}$. At low-frequencies, the corrected signal $\widehat{d}$ will accurately represent the true force measurement approximately one decade above the cut-off frequency of the complimentary filters. Therefore, accurate tracking performance is expected between $10 f_{c}$ and $f_{z}$, or the closed-loop bandwidth (whichever is lower). As shown in Fig. 4, the 3-dB closed-loop tracking bandwidth of $G_{d r}$ is $5.5 \mathrm{kHz}$.

The feedforward input $u_{f f}$ shown in Fig. 3 is used to improve the reference tracking performance of the closed-loop system. The feedforward path uses the inverse DC gain of the system as a feedforward injection filter to reduce tracking lag. To avoid exciting the high-frequency dynamics of the closedloop system, $u_{f f}$ is low-pass filtered by a 4th order Butterworth filter with $10 \mathrm{kHz}$ cut-off. The closed-loop transfer function with a feedforward input and low-frequency correction is

$$
G_{\widehat{d} r}^{f f}=\frac{k_{f f} G_{d u}+C_{d} G_{d u}}{1+C_{d} G_{\widehat{d} u}} .
$$

Fig. 4 shows the measured frequency response of $G_{\widehat{d} r}^{\text {ff }}$ with an improved 3-dB tracking bandwidth of $8.6 \mathrm{kHz}$, which is $35 \%$ of the first resonance frequency. Fig. 7 compares the tracking performance of the open-loop system, and closed-loop systems with a feedforward input. RMS tracking errors are shown in the plots.

This work is supported by the Australian Research Council.

${ }^{1}$ S. Kuiper and G. Schitter, "Active damping of a piezoelectric tube scanner using self-sensing piezo actuation," Mechatronics 20(6), 656-665 (2010).

${ }^{2}$ A. J. Fleming, "Nanopositioning system with force feedback for highperformance tracking and vibration control," IEEE/ASME Trans. Mechatronics 15(3), 433-447 (2010).

${ }^{3}$ J. L. Fanson and T. K. Caughey, "Positive position feedback control for large space structures," AIAA J. 28(4), 717-724 (1990).

${ }^{4}$ Y. K. Yong, A. Fleming, and S. O. R. Moheimani, "A novel piezoelectric strain sensor for simultaneous damping and tracking control of a high-speed nanopositioner," IEEE/ASME Trans. Mechatronics 18(3), 1113-1121 (2013).

${ }^{5}$ A. J. Fleming and K. K. Leang, "Integrated strain and force feedback for high performance control of piezoelectric actuators," Sens. Actuators, A 161(1-2), 256-265 (2010).

${ }^{6}$ Y. K. Yong and A. J. Fleming, "High-speed vertical positioning stage with integrated dual-sensor arrangement," Sens. Actuators, A 248, 184-192 (2016).

${ }^{7}$ I. Petersen and A. Lanzon, "Feedback control of negative-imaginary systems," IEEE Control Syst. Mag. 30(5), 54-72 (2010). 